\title{
INFLUENZA DELLA TORSIONE SUL MAGNETISMO DEL NIGHEL.
}

Memoria del Dott. M. CANTONE.

\section{Parte Seconda.}

Infuenza del carico tensore sui fenomeni magneto-elastici.

10. Avendo il sig. Nagaoka trovato una grande influenza del carico tensore sui fenomeni magneto-elastici ho voluto prendere in esame la questione per ricercare so con un procedimento piu sistematico di quello seguito dal predetto fisico fosse possibile fare un passo avanti in quest' ordine di studi.

Esperienze estese vennero compiute in proposito coi fili $\mathrm{Ni}$ II, $\mathrm{Ni}_{\text {II }}$ ed $\mathrm{Ni}_{\text {vm }}(3)$ durante il corse del lavoro. La variazione del carico tensore si produceva sopprimendo in alcuni casi un solo, in altri tutti e due gli anelli adattati sulla ruota: in tal guisa la tensione $\pi$ per $\mathrm{cm}^{2}$ si riduceva rispettivanente da 370 a 234 e $98 \mathrm{~kg}$.

Per queste diminuzioni, operate sempre quando il filo si trovava allo stato non deformato, I' ago del magnetometro deviava costantemente nel senso da indicare un aumento della intensiti dovuta al campo terrestre, como avea trovato l'Fwing assoggettando i fili a soli sforzi di trazione.

Col passaggio da $\pi=370$ a $\pi=234$ non si ebbero effetti notevoli sui ciclj magneto-elastici in ciascuno dei tre fili, solo nel caso di $\mathrm{Ni}_{n}$ non si ottenne per i fenomeni di accomoda. zione quella regolaritat che non mancava mai col peso tensore primitivo; ad ogrni modo si puó accertare che in generale col lavorio tende ora a manifestarsi per il magnetismo corrispondente alle forze estreme nei due sensi una piccola discimmetria.

La soppressione dei due anelli fu operata nei fili $\mathrm{Ni}_{11}$ ed Nivu(3), ma i risultati che si ebbero nei due casi furono notevolmente diversi. 'Tol primo il processo di accomodazione fu assati lungo e ridusse a ben picola cosa l' effetio delle deformazioni sui valori di M, mentro nell altro si ragginnse presto la chinsura dei cicli, ol i lenomeni magnoto-elastici si livelarono nelle condizioni finali poco attenuati rispetto a quelli avuti con un anello. 
La ragione di questo diverso comportamento parmi sia da ricercare nel latto che i fili in esame per i più piccoli valori di $\pi$ piu facilmente si mantengono diritti al crescere della plasticita della sostanza che li costituisce; e siccome il campione Nivu(3) per i tre ricuocimenti subiti era assai pastoso bisognava fare maggiore affilamento sui risultati ottenuti con esso.

Sarà bene pertanto riportare questi risultati, i quali d'altro canto si prestano meglio al nostro studio anche per la ragione che si riferiscono ad esperienze fatte nello stesso giorno usando il solo campo terpestre e cimentando il filo con ciascuno dei tre carichi tensori inclusivamente per ricerche di accomodazione l'a gli stessi limiti di ampiezza.

Aflinchè il confronto dei risultati sia reso piu agevole ho voluto segnare nella tabella che segue i valori delle letture al magnetometro non quali si ebbero, ma quali si sarebbero aruti se la declinazione fosse rimasta costante, servendomi all' uopo delle letture fatte sulla scala della bussola, che poteva benissimo servire di controllo al magnetometro per esperienze di cosi breve durata. Nella tabella figurano i valori di M e T relativi ai punti estremi di ogni ciclo ed a $\mathrm{P}=0$, non essendo richiesto dall' indole dell' attuale studio un esame particolareggiato dei cicli che niente di nuovo ci fornirebbe.

\begin{tabular}{|c|c|c|c|c|c|c|}
\hline \multirow{2}{*}{$P$} & \multicolumn{2}{|c|}{$\pi=370$} & \multicolumn{2}{|c|}{$\pi=234$} & \multicolumn{2}{|c|}{$\pi=98$} \\
\hline & M & $\mathrm{T}$ & M & $T$ & M & $\mathrm{T}$ \\
\hline 0 & $-5,2$ & $48^{\circ}, 56^{\prime}$ & $-3,4$ & $48^{\circ}, 58^{\prime}$ & $-0,8$ & $49^{\circ}, 1^{\prime}$ \\
\hline-400 & 66,1 & 12,18 & 70,5 & 12,31 & 75,2 & 12,30 \\
\hline 0 & 6,2 & 43,54 & 13,0 & 43,39 & 27,1 & 43,34 \\
\hline 400 & $-75,8$ & 85,57 & $-77,4$ & 86,24 & $-69,0$ & 86,12 \\
\hline 0 & $-16,2$ & 54,15 & $-20,2$ & 54,26 & $-20,7$ & 54,37 \\
\hline-400 & 06,5 & 12,4 & 70,3 & 12,3 & 76,4 & 12,23 \\
\hline 0 & 5,6 & 43,38 & 12,5 & 43,28 & 27,0 & 48,32 \\
\hline 400 & $-76,0$ & 87,31 & $-77,2$ & 80,24 & $-66,8$ & 86,9 \\
\hline 0 & $-17,2$ & 5,14 & $-20,6$ & 54,32 & $-28,5$ & 54,50 \\
\hline-400 & 66,7 & 12,18 & 70,0 & 12,9 & 73,9 & 12,30 \\
\hline 0 & & & & & 26,8 & 43,37 \\
\hline 400 & & & & & $-66,3$ & 86,8 \\
\hline 0 & & & & & $-27,8$ & 54,40 \\
\hline-400 & & & & & 74,9 & 12,15 \\
\hline$(0)$ & $-5,0$ & 49,3 & $-4,2$ & 48,58 & 2,8 & 49,2 \\
\hline
\end{tabular}


Dalla ispezione dei valori di $\mathrm{M}$ si vode subito quanta piccola sia l'influenza del carico tensore sulla natura dei cicli magnetici dipendenti dalle torsioni, in ispecie quando si passa da $\pi=370$ a $\pi=234$, e come neanco per le deformazioni si abbiano effetti notevoli col variare di $\pi$. Bisogna peró ricono. scere che per $\pi=98$ una dissimmetria apprezzabile si produce nell' andamento ciclico dei valori di $\mathrm{M}$, poichè in rapporto alle letture fatte al magnetometro per $\mathrm{P}=0$, tanto in principio che alla fine della serie dopo le oscillazioni, risulano numericamente più grandi le $\mathrm{M}$ che corrispondono ai pesi torcenti iniziali.

Se per il senso ora rilevato della dissimmetria vi è accordo colle ricerche del Nagaoka, per la grandezza dell' effetto non può dirsi lo stesso, giacchè cra da aspettarsi in base a queste con un carico unitario di $98 \mathrm{~kg}$. una curva trasportata tutta nel campo delle $\mathrm{M}$ positive.

Invero da noi non si operó per valori di $\pi$ crescenti, come si era fatto da quel fisico, ma non essendosi oltrepassato per tali valori i $370 \mathrm{~kg}$. non è da credere che si potessero avere condizioni elastiche diverse per un dato carico a seconda il senso del processo seguito per arrivarvi. É più probabile invece che le divergenze derivano dall' esserci nel nostro caso resi indipendenti dal magnetismo residuo, mentre nelle ricerche del Nagaoka questa causa disturbatrice senza dubbio esisteva, per cui non sarebbe azzardato l' asserire che ove nel nichel il peso tensore sia fatto variare quando il filo si trova privo di deformazioni l'influenza del carico sui fenomeni magneto-elastici, entro certi limiti, si ronde assai piccola secondo attestano i risultati della tabella VI.

\section{Influenza delle forse magnetiche sui fonomeni magneto-elastici.}

11. Molto laboriose furono le esperienze relative a questo capitolo, a causa delle complicazioni che porta l' uso di forme magnetiche piuttosto grandi, non solo pet gli offetii temporari che ne risente il tilo, ma anche, e forse di piu, per quelli residui. Però dopo molti tentativi sono riuscito a persuadermi dell' ordine con cui dorea esser condotta la ricercat ed a riconoscere le leggi del fenomeno. 
Comincio dall' intrattenermi dell' azione dei campi deboli. In tal caso per fili che avessero subito l'accomodazione l' influenza fu assai limitata qualunque fosse il senso della corrente magnetizzante, riducendoci ad avere una leggiera dissimmetria, non sempre apprezzabile, nell' andamento dei valori di M, come si puó rilevare dall' annessa tabella portante in testa alle colonne che si riferiscono alle singole serie il valore $\Delta_{B}$ della deviazione prodotta dalla corrente nella bussola; osservando che per un campo d'intensita uguale alla componente verticale terrestre occorreva che fosse $\Delta_{\mathrm{B}}=14,2$.

TAB. VIl

$\mathrm{Ni}_{11}$

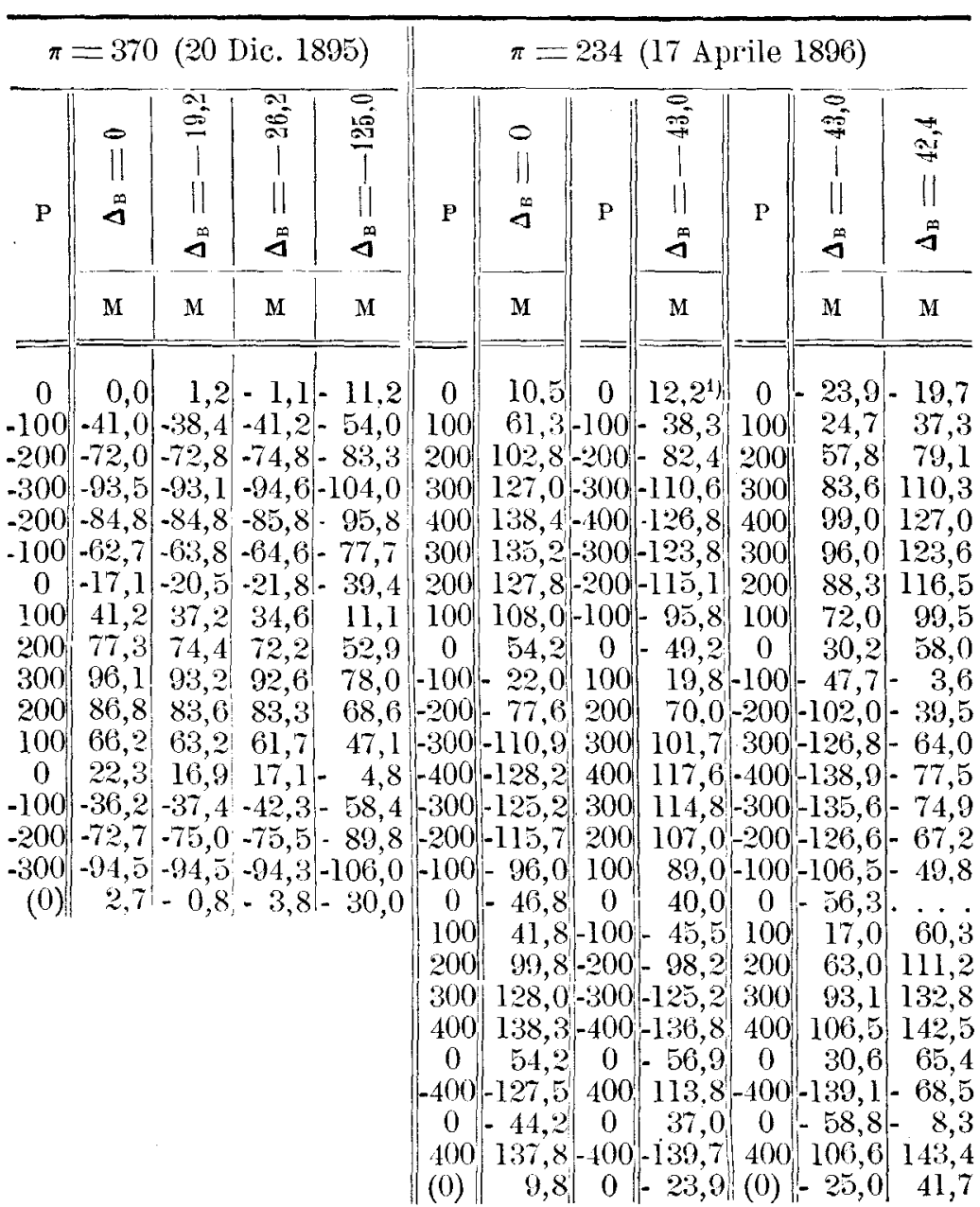

1) l'rima che passasse la co:rente or: $\mathrm{M}=15,2$. 
Questi risultati tendono a provare che il nichel, quando abbia acquistato una notevole suscettibilità a polarizzarsi colla torsione, possiede in seguito all' apparente smagnetizzazione, ottenuta col metodo noto, un assetto delle particelle che non puó essere modificato da forze magnetiche dello stesso ordine di grandezza di quelle che hanno determinato la primitiva polarita colla torsione iniziale del filo.

Ma vi ha qualche cosa di piil. Difatti anche adoperando forze magnetiche di senso opposto al campo terrestre e d' intensità nove o dieci volte più grandi, partendo sempre dallo stato non deformato, non si riesce ad ottenere che coi primi pesi torcenti s'inverta la polarita del filo per passare dal senso dovuto alla componente verticale all' altro relativo alle nuove forze magnetiche. Coi successivi cicli elastici si manifesta nel filo una marcatid discimmetria per riguardo al comportamento magnetico, e smagnetizzando in seguito il corpo colle correnti alternate il magnetometro accusa sempre una polarita residua non piccola nel senso del campo preesistente; ma non mutano per questo le condizioni di struttura interna in modo definitivo, giacchè ritornando ai cicli ordinari di deformazione sotto l' influenza del solo campo terrestre ricomparisce la simmetria, nè si hanno, col pervenire allo stato non deformato, tracce nettamente apprezzabili di magnetismo residuo.

Le proprietá magnetiche del nichel col nostro metodo di ricerca cominciano a cambiare in modo sostanziale solo con forze che sieno maggiori del limite anzidetto, cioè quando nella curva che rappresenta l' andamento delle $\mathrm{M}$ in funzione di 'T o di P la dissimmetria è tanto cresciuta che la curva stessa si trova quasi tutta da una parte dell' asse delle ascisse. Allora colle prime torsioni si esalta lo stato magnetico dovuto alla corrente sia essa cospirante od in opposizione col campo terrestre, ed alla curva ad un cappio se ne sostituisce una a due cappi.

Questo fatto si puó anche intravedere dalle ricerche del Nagaoka. Dico intravedere, poichè quantunque questo fisico abbia operato con varie forze magnetiche, pure siccome per ciascuna di esse compiva i cicli di torsione con carichi tensori crescenti, ed a misura che aumentara il campo partiva da 
valori di $\pi$ sempre più grandi, non si ha in quel lavoro materiale sufficiente per apprezzare a prima vista l'influenza delle forze magnetiche sulla legge con cui cambia la polarità del filo coi cicli di torsione; ció non pertanto dalle prime due serie si puó argomentare che con un carico di circa $400 \mathrm{~kg}$. per cm." quando si passa per la lorza magnetica dal valore 0,34 ad 1,67 nei diagrammi si va dalle curve ad un cappio a quelle a due cappi.

Del resto, per quanto sia oscura la teoria dei fenomeni magneto-elastici, il fatto di cui ei occupiamo non puó recar meraviglia, comprendendosi bene che al crescere delle forze magnetiche ha meno ragione d'essere l' anomalia del nichel, tuttora inesplicabile, dell' invertis'si della polarità col cambiar'e il senso delle deformazioni, ed aszi è da prevedere che andando a campi più intensi nei diagrammi delle $\mathrm{M}$ riferite alle $\mathrm{T}$ le curve devono tendere a divenire simmetriche rispetto all' asse delle $\mathrm{M}$.

12. Ciò è confermato dall' esperienza, e per vederlo basta passare in rassegna i risultati della tabella seguente che si riferisce a cicli di deformazione compiuti mentre nel rocchetto si avea una corrente che dava alla bussola una lettura di 408 div., ossia con una forza di circa 28 volte la componente verticale. Per i risultati che si riferiscono ad $\mathrm{Ni}_{\text {n }}$ si segnarono caso per caso i valori del peso tensore unitario $\pi$; per gli altri non si trova nella tabella alcuna indicazione in proposito, occorrerà solo avvertire che si operò per essi col carico massimo eccetto che per Nivm(3), essendosi allora avuto $\pi=98$. Per brevitá si è anche omesso di notare il senso della corrente magnetizzante, potendosi questo desumere dal segno dei valori di $\mathrm{M}$. 
INHLUENZA DFLIA TORSIONE SUL MAONET. DEL NICHEL 273

TAB. VIII

$N i_{I I}$

(Febbraio-Aprile)

\begin{tabular}{|c|c|c|c|c|c|c|c|c|}
\hline \multirow{3}{*}{$\mathbf{P}$} & \multicolumn{3}{|c|}{$\pi=370$} & \multirow{3}{*}{$\mathrm{P}$} & \multicolumn{2}{|c|}{$\pi=234$} & \multirow{3}{*}{ 更 } & \multirow{2}{*}{$\begin{array}{l}\pi=98 \\
\mathrm{M}_{0}=9,8\end{array}$} \\
\hline & $\mathrm{M}_{0}=0.4$ & $M_{0}=-7$ & $\mathrm{M}_{0}=--3,3$ & & $\mathrm{M}_{0}=15,3$ & $\mathrm{H}_{0}=13.1$ & & \\
\hline & M & M & M & & $\mathrm{M}$ & $M$ & & M \\
\hline 0 & 41,0 & $-20,3$ & $-34,3$ & 0 & 56,9 & $-26,8$ & 0 & 56,2 \\
\hline-100 & 110,2 & $-5: 9$ & $-93,0$ & -100 & 64,2 & $-16,7$ & $100^{\circ}$ & 143,7 \\
\hline-200 & 139,7 & $-127,5$ & 121,2 & -200 & 146,4 & $-127,8$ & $200^{\circ}$ & 170,3 \\
\hline-300 & 160,5 & $-154,8$ & $-161,9$ & -300 & 186,2 & $-178,8$ & 300 & 194,2 \\
\hline-400 & 185,7 & $-175,3$ & $-179,2$ & -400 & 205,6 & $-194,0$ & 400 & 203,0 \\
\hline .300 & 181,0 & $-170,3$ & $-173,0$ & -300 & 202,7 & $-101,0$ & 300 & 202,6 \\
\hline-200 & 168,1 & $-158,0$ & $-161,3$ & -200 & 193,0 & $-182,0$ & 200 & 200,2 \\
\hline-100 & 142,5 & $-133,8$ & $-136,0$ & -100 & $174,8^{\circ}$ & $-164,8$ & 100 & 195,0 \\
\hline 0 & 90,9 & $-87,2$ & $-88,4$ & 0 & 138,0 & $-131,6$ & 0 & 186,5 \\
\hline 100 & 62,9 & $-58,8$ & $-63,0$ & 100 & 127,1 & $-123,5$ & -100 & 184,2 \\
\hline 200 & 113,8 & $-107,2$ & $-123,4$ & 200 & 159,7 & $-161,8$ & -200 & 202,0 \\
\hline 300 & 162,0 & $-15 \geq, 7$ & $-160,6$ & 300 & 183,6 & $-191,3$ & -300 & 219,5 \\
\hline 400 & 179,6 & $-186,0$ & $-194,1$ & 400 & 194,8 & $-212,5$ & -400 & 230,2 \\
\hline 300 & $174,1^{*}$ & $-182,8$ & $-187,0$ & 300 & 191,8 & $-208,8$ & -300 & 228,0 \\
\hline 200 & 162,8 & $-170,8$ & $.174,2$ & 200 & 184,8 & $-199,2$ & -200 & 221,3 \\
\hline 100 & 138,7 & $-146,2$ & $.147,0$ & 100 & 164,8 & $-178,5$ & -100 & 209,7 \\
\hline 0 & 90,6 & $-93,7$ & $-90, \tilde{5}$ & 0 & 137,3 & $-138,0$ & 0 & 191,8 \\
\hline .100 & 70,3 & $-57,9$ & $-70,9$ & -100 & 134,3 & $-125,0$ & 100 & 180,2 \\
\hline-200 & 122,4 & $.105,7$ & $-116,9$ & .200 & 165,2 & $-154,5$ & 200 & 194,0 \\
\hline-300 & 165,4 & $.155,6$ & $-158,4$ & -300 & 194,7 & $-179,8$ & 300 & $205, \tilde{z}$ \\
\hline .400 & 188,3 & $-181,8$ & $.182,8$ & -400 & 209,2 & $-197,8$ & 400 & 212,0 \\
\hline
\end{tabular}




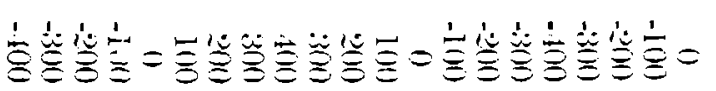

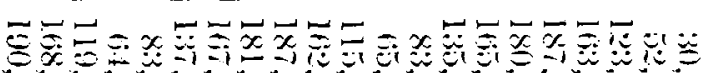

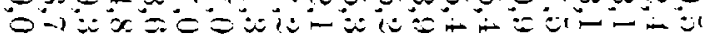

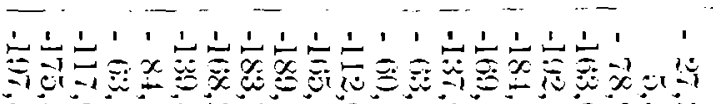

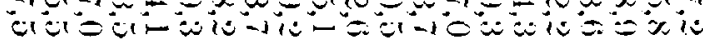

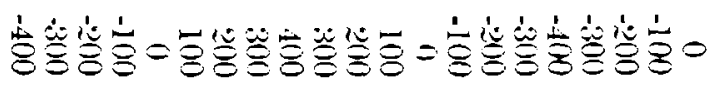

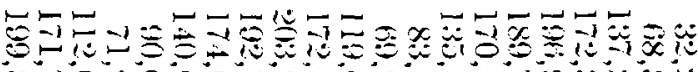
or-

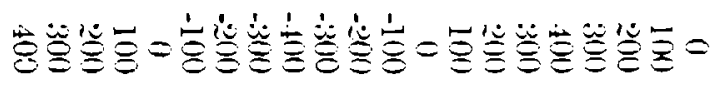
等

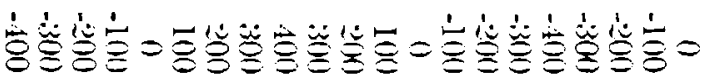

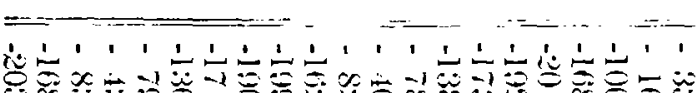

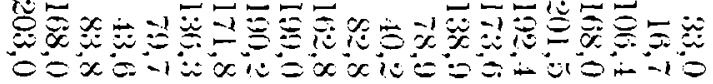

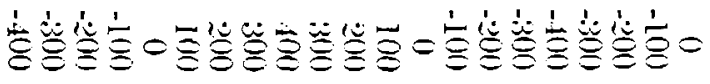

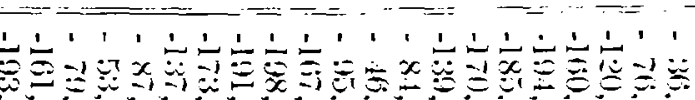

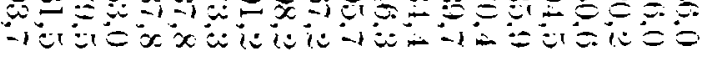

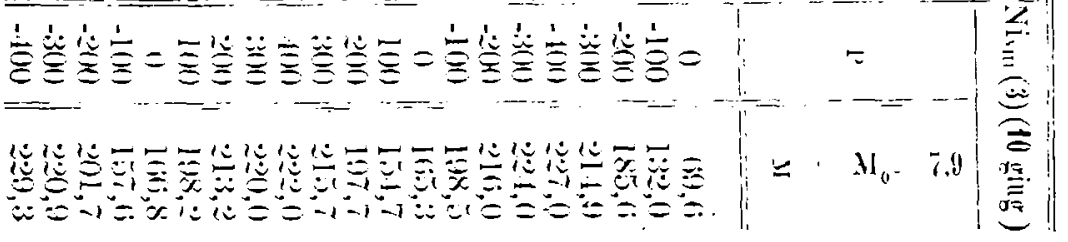

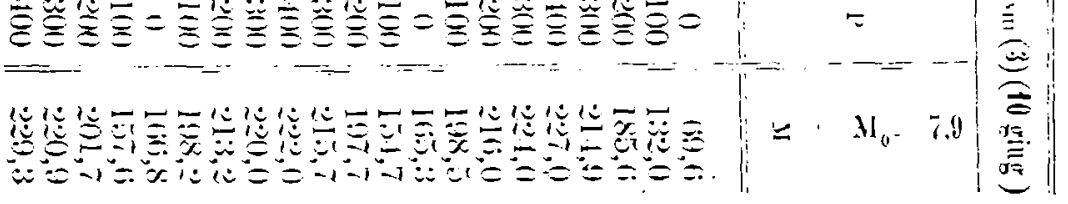


Non può sfuggire al nostro exame il fatto che restando costante il peso tensore i vari fili si comportano presso a poco allo stesso modo per i cicli magneto-clastici eseguiti in un campo magnetico piuttosto intenso, montre questo non accadeva quando agiva la componente verticale, per come si $\dot{e}$ detto al parag. 6. Si vede da ció che a misura diventano più grandi le forze magnetiche esse hanno il predominio sull'orientamento delle particelle o sullo stato di tensione del mezro che si polarizza, e che di conseguenza le perturbazioni casuali che si manifestano cosi spesso coi campi deboli piu facilmente col crescere del campo vengono meno.

Si rivela inoltre nelle serie riportate in modo notevole I'influenza dèl carico tensore nel senso di arersi una minore suscettibilita alle variazioni di M per i valori più piccoli di $\pi$, concordemente a quanto si era visto dal Nagaoka.

Molto interessante è poi il modo di comportarsi del nichel per gli effetti residui dei campi intensi che abbiano agito sopra di esso. Se a partire dalle condiżioni caratterizzate dai valori dell' ultimo rigo per ciascuna serie nella precedente - tabella vien ridotto il filo allo stato non deformato e poi si procede alla smagnetizzazione colle comenti alternate, resta nel filo una polarita resilua nel senso della forza magnetica avanti usata, ma coi successivi cicli ricomincia I'accomodazione ordinaria che porta presto nei diagranmi alle curve ad un cappio simmetriche rispetto all origine, e ciò qualunque sia il senso della torsione dalla quale ora si parte. Messo il corpo ad oscillare di nuovo non si hanno tracce notevoli di magnetismo residuo, e con ulterior'i cicli l'andamento del fenomeno non cambia di fronte alle condizioni raggiunte alla fine della serie precedente.

Questo farebbe credere a prima vista che nel filo non fossero rimasti. effelti ulteriori della intensiti generata dal campo preesistente, invece non è dificile accertare che le cose procedono in modo diverso, poichè da tutte le esperienze da me eseguite in proposito risulta che nelle ultime serie il senso della magnetizzazione per i primi valori di p non è più quello relativo al campo terrestre, l'unico che rimanga ad agrire, bensi quello che corrisponde alla forza masnetizzante per cui 
si erano avute le curve a due cappi senza inversione di polarita.

Non si puó quindi dire che per un filo già sottoposto a campi magnetici intensi si riesca coi processi dj riduzione a zero elastico e magnetico ad ottenere uno stato del tutto normale, giacchè se apparentemente con una breve accomodazione e facendolo poi oscillare una seconda volta si toglie al filo la polarità residua, questa rimane latente e si rivela nei successivi cicli ordinari per il segno dei valori di $M$ che si hanno coi primi carichi torcenti.

Da quanto siamo venuti esponendo in questo paragrafo risulta che il particolare, trovato dal Nagaoka e da noi cennato al parag. 1, sul vario orientamento della curva ad un cappio rispetto aggli assi coordinati al crescere dell' intensità del campo, a noi si palesa per via diversa. Nel nostro caso il fenomeno, secondo si è visto è spiegabile; ma non così nell'altio perchè non sappiamo quali fossero le condizioni sperimentali scelte dal Nagaoka, in ispecie per ciò che riguarda lo stato del filo quando si aumentara il carico tensore, onde non si può asserire che il particolare messo in rilievo dal Nagaoka costituisca una vera anomalia, non essendo escluso che si tratti di un fenomeno della stessa natura di quello da noi analizzato.

13. Non potevo lasciare l'argomento che forma oggetto di questo capitolo senza un esame del modo. di comportarsi del nichel sottratto all' azione del campo terrestre. Dopo quanto si era accertato sulla quasi impossibilita di avere campioni che fossero a rigore esenti da magnetismo residuo ero persuaso che, pur facendo passare per il rocchetto una corrente capace di compensare la componente verticale ed adattando in seguito il filo nell' apparecchio, coi cicli elastici qualche effetto si sarebbe apprezzato al magnetometro. Bisognava peró vedere se avendo ora da fare esclusivamente con piccole quantita di magnetismo residuo persistesse nel nichel la tendenza all' accomodazione caratterizzata dal rinforzo dei poli coi successivi cicli.

Nella tabella IX riporto i risultati cui pervenni limitandomi alle colonne delle B, P, M, e tralasciando i valori di T 
come quelli che non offrono materia a discussione nello studio attuale. Ho stimato opportuno invece indicare in testa alle colonne che si riferiscono ad ogni campione le letture $B_{0}$ ed $\mathrm{M}_{0}$ fatte alla bussola ed al magnetometro prima di mettere a posto il filo e le corrispondenti $\mathrm{B}_{1}$ ed $\mathrm{M}_{1}$ fatte appena dopo l'applicazione del peso tensore, che in queste esperienze fu sempre di $370 \mathrm{~kg}$. per $\mathrm{cm}^{2}$

TAB, IX

(27-29 Maggio)

$\pi=370$

\begin{tabular}{|c|c|c|c|c|c|c|c|c|}
\hline \multicolumn{3}{|c|}{$\begin{aligned} \mathrm{Ni}_{\mathrm{viI}} \mathrm{M}_{0}=-4,0 \mathrm{~B}_{0}=-17,0 \\
\mathrm{M}_{1}=-3,7 \mathrm{~B}_{1}=-17,0\end{aligned}$} & \multicolumn{3}{|c|}{$\begin{array}{l}\mathrm{M}_{0}=-3,0 \mathrm{~B}_{0}=-16,2 \\
\mathrm{Ni}_{1}=-1,0 \mathrm{~B}_{1}=-16,1\end{array}$} & \multicolumn{3}{|c|}{$\begin{array}{l}\mathrm{M}_{0}=-4,8 \mathrm{~B}_{0}=-17,3 \\
\mathrm{M}_{1}=-6,1 \quad \mathrm{~B}_{1}=-17,4\end{array}$} \\
\hline $\mathrm{P}$ & M & B & $P$ & M & B & $P$ & M & B \\
\hline 0 & $-4,0$ & $-17,3$ & 0 & -0.7 & $-15,8$ & 0 & $-6,2$ & $-17,8$ \\
\hline-100 & $-5,0$ & & -100 & $-1,1$ & & 100 & $-6,2$ & \\
\hline-200 & $-7,8$ & & -200 & $-0,3$ & & 200 & $-7,4$ & \\
\hline-300 & $-9,7$ & & -300 & 1,2 & & 300 & $-10,0$ & \\
\hline-400 & $-2,7$ & $-17,4$ & -400 & 3,3 & $-15,8$ & 400 & $-15,2$ & $-17,8$ \\
\hline-300 & - 2,4 & & -300 & 3,2 & & 300 & $-15,1$ & \\
\hline-200 & $-0,7$ & & -200 & 2,8 & & 200 & $-14,2$ & \\
\hline-100 & 2,5 & & 100 & 1,2 & & 100 & $-12,5$ & \\
\hline 0 & 5,2 & $-17,7$ & 0 & $-1,7$ & $-15,9$ & 0 & $-8,1$ & $-17,8$ \\
\hline 100 & 4,0 & & 100 & $-4,8$ & & -100 & $-2,1$ & \\
\hline 200 & 0,0 & & 200 & $-9,1$ & & $(-200$ & 4,5 & \\
\hline 300 & $-9,2$ & & 300 & $-15,0$ & & -300 & 12,0 & \\
\hline 400 & $-21,7$ & $-17,7$ & 400 & $-2(1,2$ & $-15,9$ & -400 & 21,2 & $-17,8$ \\
\hline 300 & $-21,7$ & & 300 & $-19,4$ & & 300 & 20,2 & \\
\hline 200 & $-21,8$ & & 200 & $-17,4$ & & -200 & 17,9 & \\
\hline 100 & $-23,2$ & & 100 & $-13,0$ & & -100 & 12,8 & \\
\hline 0 & $-22,2$ & $-17,8$ & 0 & $-4,3$ & $-15,0$ & 0 & 2,1 & $-17,9$ \\
\hline-100 & $-15,0$ & & -100 & 5,8 & & 100 & $-13,0$ & \\
\hline-200 & $-2,0$ & & -210 & 13,9 & & 200 & $-25,3$ & \\
\hline-300 & 10,3 & & -300 & 18,4 & & 300 & $-33,3$ & \\
\hline-400 & 19,6 & $-17,0$ & -400 & 21,5 & $-16,0$ & 400 & $-40,0$ & $-17,9$ \\
\hline 0 & 16,7 & $-17,9$ & 0 & $-1,2$ & $-16,0$ & 0 & $-15,0$ & $-18,0$ \\
\hline 400 & $-33,8$ & $-18,0$ & 400 & $-30,9$ & $-16,0$ & -400 & 35,0 & $-18,0$ \\
\hline 0 & $-28,2$ & $-18,0$ & 0 & $-4,1)$ & $-16,0$ & 0 & 6,0 & $-18,1$ \\
\hline-400 & 26,8 & $-18,1$ & -400 & 27,8 & $-16,0$ & 400 & $-48,7$ & $-18,2$ \\
\hline$(0)$ & -3.4 & $-18,2$ & $\|(0)$ & $-3,1$ & $-16,3$ & 10 & $-5,7$ & $-18,2$ \\
\hline
\end{tabular}




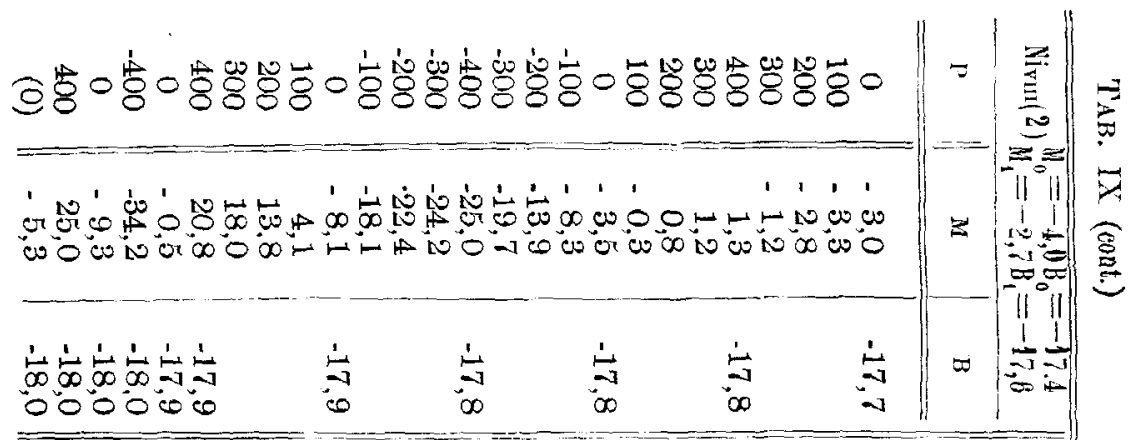

0 -

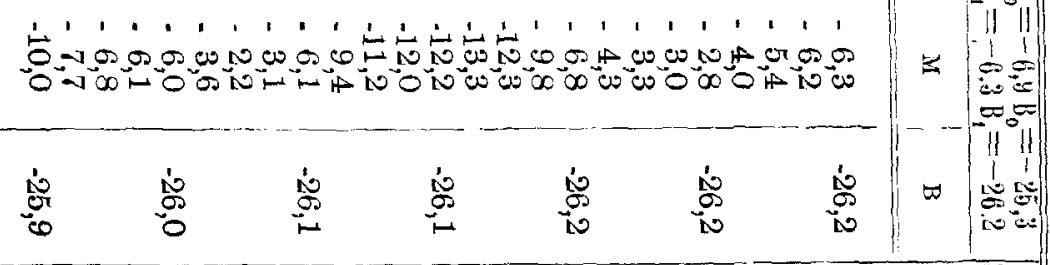

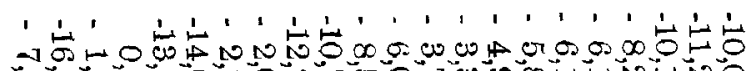
if

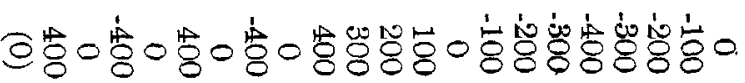

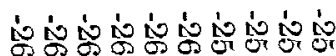
it it 000000000 है is

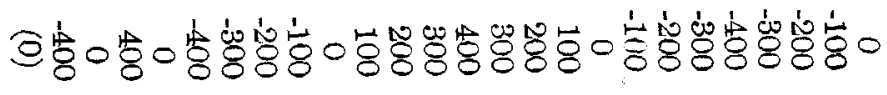

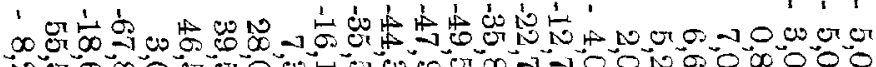

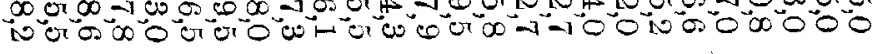

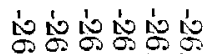
CON TON 10
0
0 $\begin{array}{ll}1 & 10 \\ 0 & 0\end{array}$

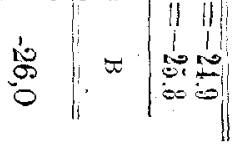


Tenuto conto della circostanza che nelle attuali esperienze i momenti magnetici acquistati dai vari fili colle prime torsioni non sono tutti dello stesso segno siamo indotti a ritenere che vi era quasi perfetto compenso fra il campo terrestre e la forza dovuta alla corrente, o che le piccole polaritì rivelate dal magnetometro quando s' introduce il filo nel rocchetto percorso dalla corrente compensatrice devono attribuirsi a cause accidentali.

Se poi esaminiamo l'andamento delle variazioni di $\mathrm{M}$ si constata come deformando il nichel vengano ad esaltarsi tracce assai esigue di uno stato magnetico spesso non bene definito in principio. Cosi ad es. il filo $\mathrm{Nivn}$, che colla introduzione nella spirale non determina alcuno spostamento dell' ago del magnetometro, colla torsione da $\mathrm{P}=0$ a $\mathrm{P}=300$ accusa nell'estremo inferiore un polo sud d' intensita crescente, se non che passando a $\mathrm{P}=400$ si inverte, almeno in apparenza, il senso della magnetizzazione e d'allora in poi comincia una vera accomodazione magnetica che procede in modo sempre più regolare a misura si va avanti coi cicli, nel senso corrispondente a quell' ultima variazione ed in guisa da aversi un aumento graduale dei ralori assoluti dei momenti magnetici estremi. Qualche cosa di analoggo accade coi campioni Nivu ed $\mathrm{Ni}_{\text {vnl }}(2)$, solo che per essi cambia il senso della variazione di M quando si passa da un peso torcente di $100 \mathrm{gr}$. ad uno di 200 gr. Invece per $\mathrm{Ni}_{1 \mathrm{x}}$ da $\mathrm{P}=400 \mathrm{a} \mathrm{P}=-300$ il lilo mostra la tendenza ad assumere all' estremo inferiore coi valori positivi di P magnetismo positivo e quello opposto quando si passa ai carichi torcenti negativi, mentre da $\mathrm{P}=-300$ a $\mathrm{P}=-400$ in luogo di ottenersi, come sempre, un aumento nel valore assoluto di $M$ si produce una diminuzione: questo è l'inizio di un processo di transizione in seguito al quale si perviene ad un andamento del tutto regolare, ma con discordanza di segno dei valori di $\mathrm{M}$ e $\mathrm{P}$, laddove in sulle prime ri era accordo.

Da tutto ció e dal vedere che non si ha una relazione costante fra i segni delle $\mathrm{P}$ e delle $\mathrm{M}-\mathrm{M}_{0}$, nè per le prime torsioni nè ad accomodazione inoltrata, si trae che le torsioni da sole non sono capaci di lare acquistare al nichel una po- 
larita mignetica; exse non possono che molificare lo stato magnetico del filo, o pel* yuesto rignamdo sono di una efficacia grandisimat, quasiche le torsioni aressero per effetto di ordinare le particelle.

Sotto tal punto di rista le ricerche magneto-elastiche darebbero un appogrgio atla teoria molecolare del magnotismo, ma resta sempre a spiegare il meccanismo per il quale nel nichel sotto l' azione di campi deboli si possa produrre per le sole torsioni una polarita opposta a quella corrispondente alla forza magnetica esterna.

Sulla legge di magnetissusione del nichel contorto.

14. Sinura non ho fornito che elementi limitati jer paragonare le variazioni dell' intensitia masnetica dovute alle torsioni colle altro provocate dalle folze marnetiche, ed in secondo luogro, nol mentre si sono studiati i cicli magnetici in rapporto alle delorn. "xioni mantenendo costante il campo, nulla abbiamo visto nel nostro erame del modo come varia l' intensita in funzione del campo lasciando costante il carico torcente. Per tali ragrioni stimo opportuno riferire su alcune esperienze fatte in quest'ordine d' ilee.

Comincio dal riportare i risultati relativi alla lege di magnetizatzione, rar cimpi crescenti, dal nichel non sottoposto a torsione, dando nella sopuente tabella i vatori di $\mathrm{B}$ e delle M che loro corrispondono, computati a partire dalle letture iniziali che si fecero per ciascuna sepie sulla scala della bussola e clel magnetometro. I M segnate, stante la piccolenza degli angoli di deriazione aruti nel magnetometro, si possono riguardare come propor\%ionali alle torye matrotizanti. Raggrimgendosi nella bussola angoli più grandi, a rendere pii

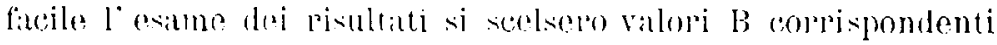

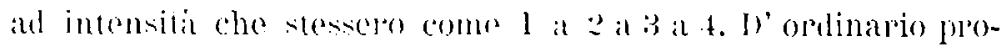

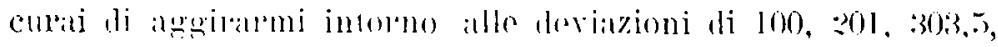
108 dive che valerano allo seoporo.

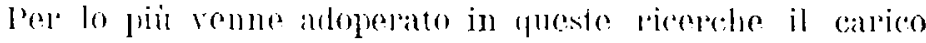

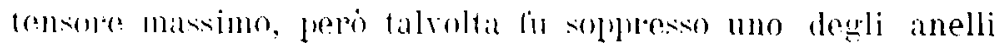


INFYUENZA DELLA TORSIONE SUL MAGNET. DEL NICHEL

ed in qualche caso resto la sola ruota, onde trovasi segnato per ogni serie il valore di $\pi$.

TAB, X

\begin{tabular}{|c|c|c|c|c|c|c|}
\hline \multirow[b]{3}{*}{ B } & \multicolumn{5}{|c|}{$N i_{11}$} & \\
\hline & \multicolumn{2}{|l|}{$\pi=370$} & \multicolumn{2}{|l|}{$\pi=234$} & \multicolumn{2}{|l|}{$\pi=98$} \\
\hline & $\begin{array}{c}25 \text { Febb. } \\
M\end{array}$ & $\mid \begin{array}{c}28 \text { Febb. } \\
\mathrm{M}\end{array}$ & ${ }_{\mathrm{M}}^{20}$ Apr & $21 \underset{\mathrm{M}}{\mathrm{Apr} .}$ & 22 Apr. & $23 \underset{M}{A p r}$ \\
\hline $\begin{array}{l}0 \\
100 \\
201 \\
303,5 \\
408\end{array}$ & $\begin{array}{r}0,0 \\
6,6 \\
13,8 \\
22,8 \\
33,3\end{array}$ & $\begin{array}{r}0,0 \\
6,8 \\
14,4 \\
22,3 \\
33,3\end{array}$ & $\begin{array}{r}0,0 \\
7,1 \\
15,6 \\
26,4 \\
40,3\end{array}$ & $\begin{array}{r}0,0 \\
7,1 \\
15,1 \\
26,3 \\
39,9\end{array}$ & $\begin{array}{r}0,0 \\
6,2 \\
14,2 \\
25,5 \\
46,0\end{array}$ & $\begin{array}{r}0,0 \\
6,4 \\
14,6 \\
25,1 \\
45,0\end{array}$ \\
\hline \multirow{2}{*}{$B$} & \multicolumn{2}{|r|}{$\pi=370$} & \multicolumn{2}{|c|}{$\mathrm{Ni}_{\mu 1}(2) n=370$} & \multicolumn{2}{|c|}{$\mathrm{Ni}_{\mathrm{VII}}(3) \quad \pi=98$} \\
\hline & $\begin{array}{c}30 \mathrm{Apr} . \\
\mathrm{M}\end{array}$ & $\begin{array}{l}30 \text { Apr. } \\
-\quad\end{array}$ & $\underset{M}{4} \underset{M}{\mathrm{Magg}}$. & $\underset{M}{4} \underset{M a g g}{\text { Magg }}$ & $11 \underset{M}{11}{ }_{M}$ Giug. & $\underset{M}{11} \underset{M}{\text { Giug. }}$ \\
\hline $\begin{array}{l}0 \\
100 \\
201 \\
303,5 \\
408\end{array}$ & $\begin{array}{r}0,0 \\
6,5 \\
\mathbf{1 3 , 3} \\
21,8 \\
\mathbf{3 2 , 1}\end{array}$ & $\begin{array}{r}0,0 \\
6,5 \\
13,3 \\
21,8 \\
31,7\end{array}$ & $\begin{array}{r}0,0 \\
6,0 \\
13,0 \\
21,0 \\
30,9\end{array}$ & $\begin{array}{r}0,0 \\
5,9 \\
13,3 \\
21,6 \\
31,5\end{array}$ & $\begin{array}{r}0,0 \\
10,0 \\
22,6 \\
40,7 \\
66,5\end{array}$ & $\begin{array}{r}0,0 \\
10,0 \\
22,6 \\
41,3 \\
66,0\end{array}$ \\
\hline
\end{tabular}

I risultati avuti per $\pi=370$ con $\mathrm{Ni}_{\mathrm{II}}, \mathrm{Ni}_{\mathrm{III}}$ ed $\mathrm{Ni}_{\mathrm{III}}(2)$ furono abbastanza concordanti, e se si tien conto che essi sono relativi a campioni differenti ed in condizioni da una serie all'altra assai diverse in rapporto ai fenomeni sinora studiati veniamo in una volta a provare la bontà della nostra disposizione sperimentale e la tendenza del nichel a conservare inalterata la sua suscettibilitá magnetica coi processi meccanici e magnetici cui si sottopone il filo, quando pero si parta dallo stato non deformato.

Pare tuttaria che un effetto del lavorio non debba mancare essendosi constatato che col campione Niu, sottoposto ad esperimento per un tempo assal lunger la suscettibilita presentò nei primi due mesi un abbansamento semsibile, come si

Serie $I V$, Tol. $r$. 
può argomentare confrontando i risultati della precedente tabella con quelli contenuti nella seconda colonna della tab. XI, senza d'altra parte aver ragione di credere che si Cossero modificate in questo intervallo le condizioni del magnetometro.

Apparisce altresi dalla tab. $\mathrm{X}$ una influenza del carico tensore sulla legge di magnetizzazione del nichel per il variare del campo, nel senso indicato già dall' Ewing, avendosi per lo stesso valore della forza intensità crescenti al diminuire del peso tensore.

15. Per il nichel contorto i risultati sono di tutt'altra natura; essi si discostano dai precedenti nel senso di avvicinarsi a quelli avuti dal Nagaoka in ricerche del medesimo genere operando con forti deformazioni; nelle mie esperienze vi è peró qualche particolare nuovo che credo utile porre in rilievo servendomi delle tabelle XI, XII e XIII.

1)i tali esperienze la parte relativa alla tabella XI fu fatta nel seguente modo. Il filo reso privo di deformazioni fu sottoposto prima ad un ciclo magnetico fra i valori limiti di B 408 e - 408, e poi a cicli della stessa ampiezza ma sotto l'azione dei carichi torcenti segnati in testa alle successive colonne a partire dalla terza. Il processo di smagnetizzazione attuato fra una serie e l'altra qui non fu in generale efficace, come si puó rilevare dai valori di $\mathrm{M}$ che trovansi in fondo a ciascuna colonna assieme alle $B_{0}$ indicanti le letture fatte in corrispondenza sulla scala della bussola.

In condizioni diverse furono condotte le esperienze che si riferiscono alla tab. XII, in quanto allora i cicli magnetici furono eseguiti sotto l'azione di un peso torcente - 400 cui si era giunti dopo un ciclo elastico fra $\mathrm{P}=-400 \mathrm{e} \mathrm{P}=400$.

Inline la tabella XII contiene i risultati di un ciclo magnetico compiuto col filo $\mathrm{Ni}_{m}(2)$ e con un carico torcente di $550 \mathrm{gr}$. in seguito alle esperienze di cui si parlò al $\$ 9$. 
IN FLUENZA DELLA TORSIONE SUL MAGNET. DEL NICHEL

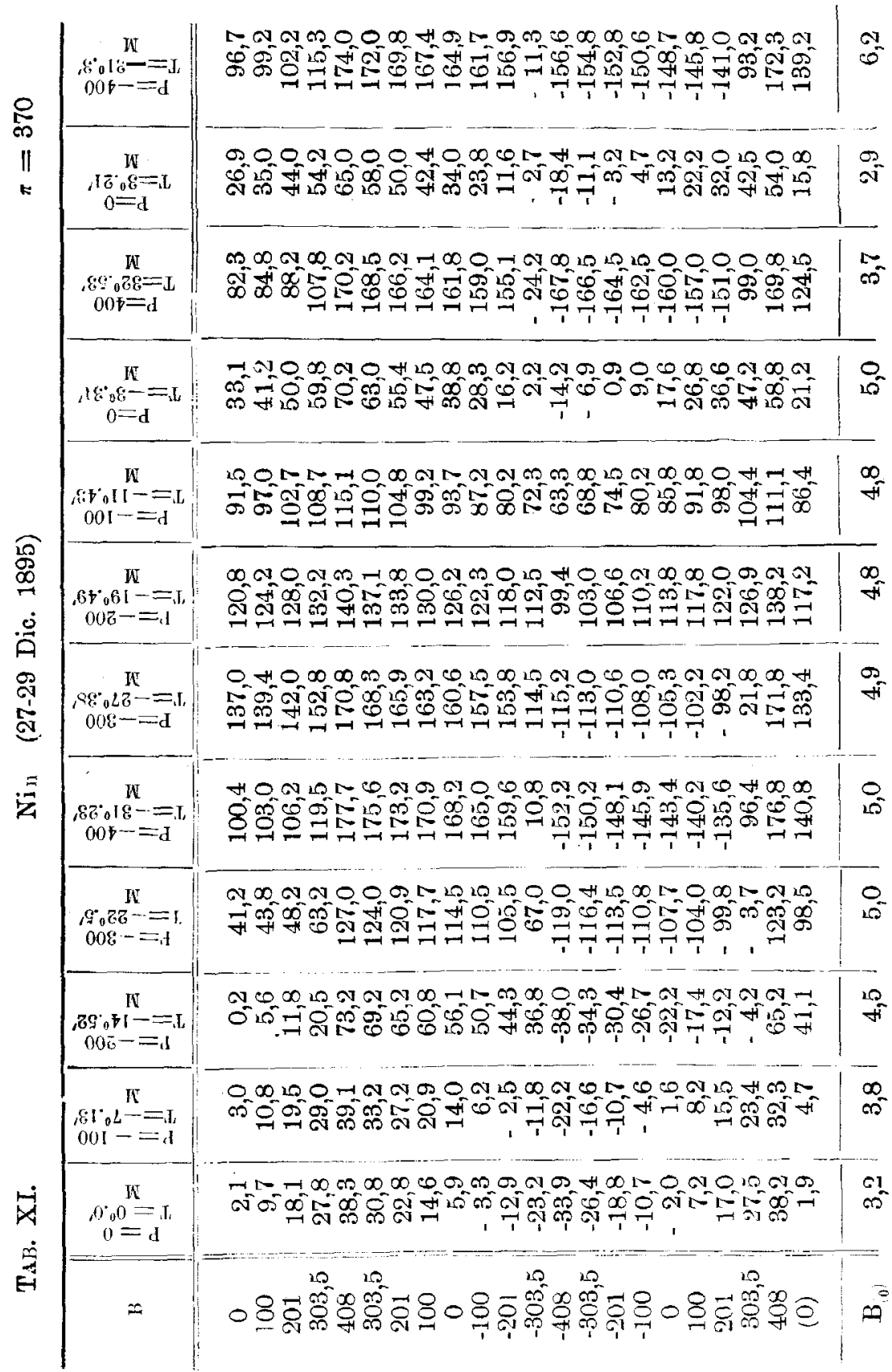


TAB. XII

$\mathrm{Ni}_{\text {II }}$ (22 Genn. 96)

$\pi=370$

\begin{tabular}{|c|c|c|c|c|c|c|c|}
\hline $\mathrm{P}$ & B & M & $\mathrm{T}$ & $\mathrm{P}$ & B & $\mathrm{M}$ & $\mathrm{T}$ \\
\hline $\begin{array}{c}0 \\
-100 \\
-200 \\
-300 \\
-400 \\
-300 \\
-200 \\
-100 \\
0 \\
100 \\
200 \\
300 \\
400 \\
300 \\
200 \\
100 \\
0 \\
-100 \\
-200 \\
-300 \\
-400\end{array}$ & 6,6 & $\begin{array}{r}4,9 \\
62,7 \\
103,2 \\
125,1 \\
135,2 \\
130,5 \\
119,0 \\
92,9 \\
33,8 \\
-49,8 \\
-100,2 \\
-125,2 \\
-134,5 \\
-129,2 \\
-116,2 \\
-89,8 \\
-27,8 \\
57,2 \\
104,3 \\
126,3 \\
135,5\end{array}$ & $\begin{array}{r}0,0 \\
-7,31 \\
-14,55 \\
-22,33 \\
-31,4 \\
-26,9 \\
-18,53 \\
-10,54 \\
-2,35 \\
5,45 \\
13,52 \\
22,50 \\
31,38 \\
25,53 \\
18,13 \\
10,49 \\
2,28 \\
-5,55 \\
-13,46 \\
-22,13 \\
-30,48\end{array}$ & 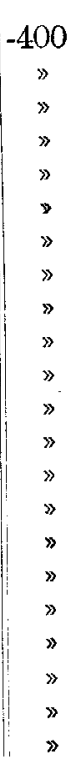 & $\begin{array}{r}6,8 \\
107,0 \\
208,0 \\
311,0 \\
415,0 \\
311,0 \\
208,0 \\
107,0 \\
6,6 \\
-94,0 \\
-193,0 \\
-297,0 \\
-402,0 \\
-297,0 \\
-193,0 \\
-94,0 \\
6,4 \\
106,0 \\
207,0 \\
310,0 \\
415,0 \\
7,31,\end{array}$ & $\begin{array}{l}135,5 \\
138,7 \\
142,8 \\
163,2 \\
174,7 \\
172,4 \\
169,9 \\
167,6 \\
164,8 \\
161,7 \\
156,4 \\
-22,0 \\
-155,0 \\
-153,0 \\
-150,8 \\
-149,7 \\
-146,2 \\
-143,2 \\
-138,0 \\
100,0 \\
173,2 \\
141,8\end{array}$ & 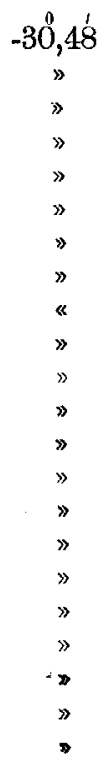 \\
\hline
\end{tabular}

TAB. XIII $\mathrm{Ni}_{m}(2)$ (6 Maggio) $\mathrm{P}=-550 \mathrm{~T}=70^{\circ} 0$

\begin{tabular}{c|c||c|c}
\hline $\mathrm{B}$ & $\mathrm{M}$ & $\mathrm{B}$ & $\mathrm{M}$ \\
\hline 0 & 105,3 & 303,5 & 147,0 \\
-100 & 103,3 & 408 & 188,0 \\
-201 & 83,0 & 303,5 & 188,6 \\
$-303,5$ & $-143,8$ & 201 & 188,8 \\
-408 & $-194,5$ & 100 & 188,8 \\
$-303,5$ & $-195,0$ & 0 & 188,7 \\
-201 & $-195,0$ & -100 & 188,6 \\
-100 & $-195,0$ & -201 & 182,8 \\
0 & $-194,2$ & $-303,5$ & $-46,0$ \\
100 & $-192,2$ & -408 & $-191,0$ \\
201 & $-187,2$ & &
\end{tabular}

1) Si ritorna alla forza marnetizzante zero col motodo delle correnti alternato decresecenti. 
Occupiamoci prima della tab. XI. ì da notare anzitutto quanto poco sia altelata la legge di magnetizzazione del nichel da $\mathrm{B}=0$ a $\mathrm{B}=408$ ed anche lungo il ciclo successiro pas. sando dalla prima serie compiuta nelle condizioni normali alla seconda eseguita con un peso torcente di - $100 \mathrm{gr}$., ed il fatto è tanto più rimarchevole per ció che venendo da $\mathrm{P}^{\prime}=-100$ a $\mathrm{P}=-200$ una sensibile modificazione si presenta nelle proprietá magnetiche del lilo, avendosi, rispetto alle condizioni primitive, un grande innalzamento della suscettibilita quando ci si avvicina ai punti estremi del ciclo, ed una diminuzione quando da questi estremi ci si allontana. Per $\mathrm{P}=-300$ l'influenza della deformazione sul fenomeno magnetico, si rende ancora piu forte, e finalmente per $\mathrm{P}=-400$ l'isteresi magnetica è tanto cresciuta che renendo la prima volta da $B=408$ a $\mathrm{B}=-408$ la $\mathrm{M}$ per $\mathrm{B}=0$ si trova diminuita del cinque per cento, e non cambia segno se non per $\mathrm{B}=-408$ : e nel passaggio da $\mathrm{B}=-408$ a $\mathrm{B}=408$ i ralori di $M$ procedono presso a poco colla stessa legge che per la prima meta del ciclo.

Riducendo a - 300 il peso torcente tendono a mantenersi esagerati gli effetti della torsione sulla isteresi magnetica del filo, ma rendendo in seguito ancora piu piccolo il valore assoluto di $P$ il fenomeno cambia natura talmente che per $\mathrm{P}=-100$ si può dire sparita l'isteresi. La si rede ricomparire quando si fa $\mathrm{P}=0$, ed allora la legge di magnetizzazione ad un dipresso quale si era avuta per i risultati della seconda colonna.

Ja questo andamento è facile argomentare ció che deve accadere per i valori di $\mathrm{P}$ da zero a 400 e da 400 a - 400, sicchè mi son limitato ad esporre nelle ultime tre colonne i risultati riferentisi a $\mathrm{P}=400, \mathrm{P}=0 \mathrm{e} \mathrm{P}=-400$ per mo- $^{\mathrm{P}}$ strare come colle delormazioni estreme i ralori di $\mathrm{M}$ che trovansi nello stesso rigo sieno assai concordanti fra loro, e con quelle permanenti si modifichi poco, rispetto ai risultati della seconda colonna, la legge con cui variano le $\mathrm{H}$ al variare di B, prescindendo, bene inteso, dal magnetismo residuo che in questo caso affetta in modo diverso le $\mathrm{M}$ a seconda si giunga a $\mathrm{P}=0 \mathrm{da} \mathrm{P}=-400$ o.da $\mathrm{P}=400$. 
Faró osservare che alla fine delle esperienze cui si riferisce l'ultima colonna il filo fu ricondotto mediante le oscillazioni allo stato non deformato, ed allora i valori di $\mathrm{B}$ ed $\mathrm{M}$ furono rispettivamente di 6,9 e 3,8 , mentre si era partiti al 27 dicembre da $\mathrm{B}=3,7$ ed $\mathrm{M}=3,1$. Si vede pertanto che al termine della serie risultó poco variata, in paragone alla primitiva, la intensitid magnetica del filo, non ostante i processi magnetici ed elastici cui fu sottoposto il nostro campione. Anzi deve aggiungersi che qui si è riusciti a togliere il magnetismo residuo senza bisogno di ulteriori cicli di torsione, mentre d'ordinario partendo da $\mathrm{P}= \pm 400$ e da $\mathrm{B}= \pm 408$ allo stato nolmale non si arriva senza questo ulteriore lavorio avendosi in seguito ai due metodi di piduzione a zero magnetico ed elastico una polarità residua nel senso che corrisponde all' ultima delle forze magnetizzanti estreme. Pare dunque che le variazioni cicliche di $B$ coi diversi carichi torcenti in un senso e nell' altro, e di prefercnza coi pesi estremi, abbiamo avuto per effetto nelle esperienze relative alla tabella XI di fare sparire colla smagnetizzazione ogni traccia di polaritá che non tosse dovuta alla deformazione, per modo che, tolta questa, il filo è potuto tornare direttamente allo stato normale.

Nelle esperienze del 22 Gennatio, come si disse, lo studio venne fatto solo per $\mathrm{P}=-400$; tuttavia $\mathrm{i}$ risultati sono di un certo interesse, poichè presentano un accordo discreto con quelli che si ebbero per lo stesso peso torcente nella tab. XI, non ostante che a questo si fosse giunti allora con un processo più complicato. Si trae da ciò che in un filo contorto notevolmente l'assetto delle particelle, per quanto riguarda la suscet. tibilita a polarizzarsi sotto l'azione delle forze magnetiche, pur essendo diverso che nello stato primitivo si presenta assai stabile, nè può essere gran fatto modificato dai processi intermedi magnetici od elastici.

I risultati dell' ultima tabella ci mostrano il comportamento magnetico del filo $\mathrm{Ni}$ (2) sottoposto a deformazione esagerata. I valori che in tali condizioni si raggiunsero per l'intensitá non furono molto maggiori di quelli ottenuti con $\mathrm{Ni}_{1}$ con un peso torcente di $400 \mathrm{gr}$; ma abbiamo che ora nel ritorno dai punti estremi del ciclo si produce in luogo di una diminuzione 


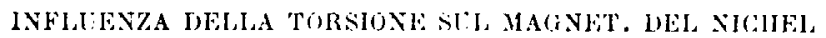

un aumento nel valore assoluto dell' intensiti, sicchè con $\mathrm{B}=0$ si hanno nei due passagrgi da $B \ldots 408$ a $B=408$ e da $B=408$ a $B=-408$ ralori di $\mathrm{X}$ o uguali o numericamente più grandi che nei punti estremi del ciclo. 'Trattandosi di un' anomalia che si palesa in propor\%ioni limitate non è il caso di redere quali possano essere le cause che la determinano. ci contenteremo di con-tatite che in un lilo il quale abbia subito gli scorrimenti continui, gli offetti della isteresi magnetica riescono ancora più rileranti di quelli messi in luce dal Nagraoka nelle sue ricerche.

Inuate il corso dolle esperienze che si riteriscono al palag. a ho aruto il mazo di accertare che azioni sussegrlenti magnetiche si producono in generale quando interviene elasticità di seconda specie. Peroi nello stuelio della legge di magnetizzazione del nichel contorto ho potuto riconoscere che altre ne esistono quando il lapporto tra le variazioni di the di $B$ é assai grande, ma yui io credo siamo di fronte al un fatto dipendente non dallo stato di deformazione bensi la una instabilita nell assetto magnetico del filo. Noi abbiamo gia risto che con fili tortemente deformati nel passiagyio da una forza magnetica estrema all altra il corpo presenta in principio la tendenza a conserrare la polarita per invertirla in ultimo di un tratto, onde siamo autorizzati a ritenere che nei punti ricini a questo stato critico, non si possa avere equilibrio magnetico stabile, e siccome allora si rerilicano per le leture al magnetometro, forti arioni susseguenti, la loro esistenza pare debba attribuirsi a quella instabiliti.

16. I risultati della tab. XI, per la parte che riguarda le colonne $2^{\mathrm{a}}, 3^{3}, 4^{\mathrm{a}}, 5^{\mathrm{a}}$ e $6^{\mathrm{a}}$ mostrano come a misura si torce il dilo cambia la legre che segue il corpo nel magnetizarsi; pero si comprende che non puó mancare in ciascuno dei cicli, a fartire dal terzo, l'influenza del mignetismo residuo; restara percio a redere se ri fosse il mezzo di orvialo a questo inconveniente per uno studio piu accurato del fenomeno in exame.

Mi sono convinto che per riuscire allo scopo conreniva partire dallo stato del filo per cui losse $\mathrm{I}= \pm 108$ e $\mathrm{P}= \pm 400$, 
attuare poi i due metodi di riduzione a zero, ritornare ai cicli elastici fra $\mathrm{P}=400$ e $\mathrm{P}=-400$, e porre in ultimo il filo ad oscillare. Con questo processo preliminare che chiamero di adattamento era possibile di ottenere volta per volta stati del filo paragonabili fra loro.

I risultati avuti con queste cautele furono assai soddisfacenti per l'accordo che si ebbe in tutti i casi f'ra $i$ valori iniziali di M, e per' l' accordo che presentavano le serie di esperienze fatte a lunghi intervalli di tempo colla stessa deformazione, comunque fosse rariata dall' una all' altra di queste serie il magnetismo del filo vuoi per le forze elastiche vuoi per le magnetiche.

Ciò mi spinse ad estendere il campo di ricerca per esaminare l'influenza del carico tensore sui cicli magnetici, operando ora con due anelli, ora con uno, ora col solo peso della ruota colla puleggia.

Le ricerche, eseguite col filo $\mathrm{Ni}_{\mathrm{Ix}}$ (4), procedettero nel seguente modo. Prendendo le mosse dal carico tensore massimo ed attuato una prima volta il processo di adattamento si compirono due cicli magnetici col filo non deformato fra i valori di B 408 e - 408. Ripetute poi le operazioni preliminari come nel caso precedente, si produssero altri due cicli magnetici fra i medesimi limiti di $\mathrm{B}$ col filo deformato dal carico torcente di $100 \mathrm{gr}$., e cosi di seguito usando volta per volta valori di $P$ più grandi. Allo stesso modo vennero condotte le successive esperienze con un anello e le altre col carico tensore minimo.

Si trovó che al diminuire di $\pi$ cresceva sempre l'intensità magnetica del filo nel senso corrispondente al campo magnetico terrestre, in modo poco marcato passando da $\pi=370$ a $n=234$, e notevolmente quando si venne a $\pi=98$.

Si vide inoltre che anche con questo carico il processo di adattamento era del tutto efficace permettendo di ottenere in ogni serie valori iniziali di M pressochè costanti.

Gli annessi diagrammi rappresentano i risultati ottenuti. Ogni curva corrisponde ad un ciclo magnetico eseguito sotto l' azione della forza torcente che trovasi indicata nella figura (Tav. III) e serve ad indicare la legge con cui varia la $\mathrm{M}$ in funzione di $\mathrm{B}$. 
L' esame dei diagrammi ci rivela subito quanto grande sia l' influenza delle deformazioni sui cicli magnetici. Questa influenza si manifesta sia collo spostarsi del centro di simmetia della curva che rappresenta il ciclo, sia col crescere dell' area racchiusa dalla curva stessa. Quando si passa da $\mathrm{P}=0$ a $\mathrm{P}=100$ non si produce che il primo fatto, invece venendo a $\mathrm{P}=200$ è assai marcato il secondo, e ciò si verifica per ciascuno dei tre diagrammi.

Riguardo al carico tensore si trova che al diminuire di $\pi$ cresce I' area d'isteresi senza d' altra parte aversi un notevole spostamento del centro di simmetria, ed infine che l'aumento che si produce nell' area al diminuire di $\pi$ apparisce meno evidente a misura si va a valori più elevati di $P$.

\section{Infuenza del magnetismo sulle deformazioni.}

17. Poco ho da dire su quest' ultimo argomento, giacchè dalle numerose esperienze da me fatte in tutto il corso del lavoro risulta che, mentre le deformazioni influiscono in modo notevole sullo stato magnetico del filo, il fatto reciproco non si palesa, essendosi potuto constatare che per cicli elastici com. piuti col campo magnetico e le deformazioni estreme nei due sensi e le aree d'isteresi non vengono modificati rispetto ai valori avuti senza il campo; sicchè parmi sia da concludere che, se esiste un' influenza dei cicli magnetici su quelli elastici, dev' essere di un ordine di grandezza assai piccolo.

\section{RIEPILOGO.}

Riassumo i risultati principali cui sono pervenuto in questo lavoro.

$1^{0}$ Coi cicli di torsione compiuti lpa determinati limiti di forze estreme, sotto l' azione del campo terrestre e con un peso tensore di $370 \mathrm{~kg}$. per $\mathrm{cm} .{ }^{2}$, s' inverte la polarità dei fili di nichel al cambiare di segno della forza deformatrice, e d'altra parte i-valori assoluti dei momenti magnetici quando si giunge ai punti estremi del ciclo vanno crescendo col lavorio del corpo in modo assai marcato in principio e colla 
tendenza in ultimo ad un andamento stazionario, in guisa da rilevarsi un processo di accomodazione capace di esaltare considerevolmente la suscettibilita del nichel a polarizzarsi colla torsione colla legge indicata dal Nagaoka.

$2^{0}$ Hacendo oscillare il filo a partire da una delle deformazioni estreme cui si perviene nei cicli anzidetti si riesce a togliere al filo, almeno in apparenza, ogni traccia di polarita residua; il che permette di disporre di uno stato normale per le serie successive senza bisogno di ricorrere all' arroventamento, che del resto modificherebbe le condizioni elastiche del corpo in esame.

$3^{0}$ Se prendendo le mosse da una forza estrema si ritorna ad essa dopo essere giunti al carico torcente zero, o ad uno uguale in valore al primo e di segno opposto, i cicli magne. tici offrono una grande analogia con quelli elastici. $\dot{\mathrm{E}}$ peró da notare che nel secondo caso, mentre le curve che rappresentano l'andamento delle intensità in funzione dei pesi torcenti risultano tutte tracciate nel senso degli indici dell' orologio, quando invece nei diagrammi delle intensità stesse si assumono come ascisse gli angoli di torsione per piccoli valori estremi, di queste il senso rimane inalterato, ma una inversione si produce venendo alle ampiezze più grandi. $\dot{\mathbf{E}}$ da argomentare pertanto, e le nostre esperienze lo mostrerebbero sino ad un certo punto, che esista un valore dell' ampiezza per il quale nelle variazioni delle intensità in funzione degli angoli non si ha isteresi.

$4^{0}$ A partire dallo stato non deformato col crescere dei pesi torcenti aumenta l'intensità magnetica del nichel, ma se si eccede nelle torsioni il momento magnetico diminuisce sino a raggiungere un limite al di sotto del quale pare non si rada, anche nel caso in cui non sono più possibili deformazioni stabili del corpo. Il cambiamento di segno nelle variazioni dell' intensita magnetica si verifica quando gli effetti della elasticita di seconda specie cominciano a diventare notevoli: questo particolare e la circostanza di aversi sempre per forze decrescenti una diminuzione nel valore assoluto della intensità fanno pensare che i fenomeni magneto-elastici non dipendano dalle 
semplici deformazioni, ma che debbano forse entrare in giuoco anche le reazioni elastiche.

$5^{0}$ Si hanno due specie di azioni susseguenti maguetiche: le une si manifestano nei casi in cui interviene elasticita di seconda specie; le altre, producendosi quando nei cicli magnetici s'inverte la polaritá del filo ed il rapporto fra le variazioni dell' intensitá e della forza magnetica assume valori assai grandi, pare dipendano da un assetto magnetico instabile delle particelle.

$6^{0}$ Se si fa diminuire il carico tensore mentre il corpo si trova nello stato non deformato, e se la cederolezza del nichel permette che l' asse del filo si mantenga diritto anche coi carichi più piccoli, l'effetto dello stiramento, nei limiti entro i quali furono estese le nostre esperienze, è poco rimarchevole limitandosi ad una leggiera dissimmetria nella curva ad un cappio che rappresenta il ciclo magnetico. Il disaccordo che si avrebbe per tal riguardo nei risultati quantitativi colle ricerche del Nagaoka deriva forse dal tatto che nelle esperienze di questo fisico si facea sentire l'influenza del magnetismo residuo.

$7^{0}$ Forze magnetiche dello stesso ordine di grandezza del campo terrestre, agenti in un senso o nell'altro su di un filo gia accomodato pere i cicli magneto-elastici sotto l'azione di questo campo, non modificano la natura dei cicli magnetici dovuti alle torsioni in maniera nettamente apprezzabile nè per gli effetti temporari nè per quelli residui. Andando a forze più grandi una dissimmetria si produce nella curva che rappresentä il ciclo, e solo quando si perviene a forze che sieno più del decuplo della componente verticale esse prendono il predominio sul fenomeno, nel senso che la polarita dipende nelle nuove condizioni dal campo attuale, ed allora alla curva ad un cappio si sostituisce quella a due cappi. In tal caso smagnetizzando il filo colle correnti alternate e decrescenti e facendolo poi oscillare si ha magnetismo residuo che sparisce in apparenza coi successivi cicli elastici e con una nuova serie di oscillazioni. Ma con ulteriori torsioni la polarita che assume il filo quando si va per la prima volta allo sforzo torcente massimo è nel senso del forte campo preesistente. 
$8^{0}$ Il magnetismo che è capace di acquistare il nichel per forze magnetiche 18 volte pia grandi del campo terrestre, è appena la quarta parte di quello che si può ottenere d'ordinario sotto l'azione della componente rerticale torcendo il filo di $l^{0}$ per $^{2} \mathrm{~cm}$.

$9^{0}$ La legge di magnetizzazione del nichel contorto per forze magnetiche le quali variino in modo ciclico cambia no. terolmente col peso tercente. L' area d' isteresi cresce coll'iumentare di questo, e diminuisce inrece quando si opera con forze elastiche decrescenti, colla tendenza ad annullarsi prima che si arrivi in tale serie di cicli a quello che corrisponde al peso zero. Colle deformazioni permanenti la legge che segue il corpo nel magnetizzarsi al variare del campo è poco modificata rispetto all' andamento che presentano le intensitì nei cicli magnetici compiuti col filo non deformato, ma si sposta il centro di simmetria della curva figurativa del ciclo a causa del magnetismo residuo. Siffatta influenza non si palesa coi carichi torcenti estremi in un senso o nell' altro: in tal caso le curve relative ai due cicli magnetici eseguiti con questi carichi risultano quasi coincidenti, avendosi cosi, come era facile aspettarsi, effetti delle deformazioni che dipendono dalla grandezza di queste e non dal loro segno.

$10^{\circ}$ I risultati che si riferiscono al n. $9^{\circ}$, per la parte che riguarda i cicli magnetici eseguiti con forze di torsione crescenti, si rendono meno complicati se con un processo facile ad attuarsi si parte volta per volta da condizioni del filo paragonabili fra loro, venendo meno in tal guisa l' influenza del magnetismo residuo, e collo stesso artifizio si ha il modo di apprezzare nel suo giusto valore l' influenza del carico tensore sulla suscettibilità del nichel contorto.

$11^{0}$ Coi mezzi di misura di cui disponiamo non si è potuto riconoscere nelle attuali ricerche se le forze magnetiche sieno capaci di modificare le proprietà elastiche del filo. Ciò è probabile dipenda dal fatto che le tensioni dorute a queste forze sono assai piccole di fronte a quelle prodotte dalle ordinarie deformazioni.

Laboratorio di fisica della R. Uuiversità di l'ilermo, Gennaio 1897. 